\title{
ARTICLES
}

\section{Degenerate Blume-Emery-Griffiths model for the martensitic transformation}

\author{
Eduard Vives and Teresa Castán \\ Department d'Estructura i Constituents de la Matèria, Universitat de Barcelona, \\ Diagonal 647, E-08028 Barcelona, Catalonia, Spain \\ Per-Anker Lindgård \\ Department of Condensed Matter Physics, Ris $\phi$ National Laboratory, DK-4000 Roskilde, Denmark
}

(Received 30 October 1995)

\begin{abstract}
A generalization of the Blume-Emery-Griffiths model is introduced in which an entropy stabilization of the high-temperature phase is controlled by a degeneracy parameter $p \geqslant 1$. The model describes a first- and a second-order phase transition as a function of temperature between two ordered phases. This is relevant for the martensitic transition problem. Mean-field calculations and Monte Carlo simulations are presented. The model predicts a constant entropy change at the transition for various transition temperatures in agreement with the behavior found experimentally.
\end{abstract}

\section{INTRODUCTION}

Many crystals, with examples ranging from simple metals to complicated, technologically useful alloys and ceramics, undergo a structural phase transition from a close-packed (CP) structure at low temperature to an open body-centeredcubic (bcc) structure at higher temperatures. This is a generic feature of the so-called martensitic transition. ${ }^{1}$ The stability of the open structure is associated with a large entropy ${ }^{2,3}$ for that phase. There is some electronic contribution. However, we shall neglect this here since it is known from firstprinciples calculations ${ }^{4,5}$ and experimentally ${ }^{6,7}$ to be smaller than the "vibrational part." That part can only in the crudest approximation be described by a quasiharmonic phonon picture, since it is known that the relevant phonons are strongly damped or even overdamped. ${ }^{6,8}$ The transition is displacive, diffusionless, and weakly discontinuous, usually with negligible associated change in volume. It should, therefore, be well suited for a description in terms of lattice spin models.

A continuous spin model was proposed by Lindgård and Mouritsen $^{9}$ (LM) in order to describe the transition between two ordered phases. This fairly closely follows the physics involved in the idealized martensitic transition from the hcp to the bcc structure found in $\mathrm{Zr}$. Monte Carlo simulations showed large heterophase fluctuations, the entropy contribution of which is not well described by quasiharmonic theory as discussed in Ref. 10. In a further study by Castán and Lindgård ${ }^{11,12}$ several simplifications were proposed. Here we shall demonstrate that the models may be simplified even further to the class of the three-state Blume-Emery-Griffiths (BEG) model. ${ }^{13}$ We shall generalize this model to include a whole family of simple multiple-state models with the property that there exists an entropy-stabilized high-temperature phase separated by a continuous or discontinuous phase transition from the low-temperature phase. We will study the general properties of these models by means of mean-field theory and Monte Carlo computer simulations.

Another lattice gas model explicitly including vibrational entropy effects has been proposed by Morris and Gooding. ${ }^{14}$ It has recently been used to test a quasiharmonic approach to determine the entropy from simulations. ${ }^{15}$ The model stabilizes the high-temperature phase by lowering the vibrational entropy of the low-temperature phase. This is done by means of an anharmonic interparticle coupling which has almost no effect on the high-temperature phase but, at low temperatures confines the particles so that large amplitude vibrations are inhibited. The symmetry-breaking term is local, and therefore this model has more restricted statistical properties than a pair interaction spin model.

We shall here introduce the entropy stabilization in a much simpler way than discussed in the previous models. The martensitic phase has several low-temperature variants, i.e., degenerate phases. These could describe the displacements in the $\pm x, \pm y$, or $\pm z$ direction, respectively, leading to six variants. The high-temperature phase corresponds to the phase with zero average displacement, i.e., $x=y=z=0$. A system in this state may choose any of the six displacements, and can thus be thought of as having a high degeneracy with respect to large amplitude vibrations. In a given low-temperature phase there is already a chosen direction, for example, $x$, and the degeneracy is only 2 , namely, $\pm x$. There is a very large energy barrier for going from these states to one of the other possible variants or domains $\pm y$ and $\pm z$ due to strain effects (not explicitly included in the model). This is the physical picture behind the generalization of the BEG model, which is discussed more in the Appendix. In the original BEG model ${ }^{13}$ the 0 -phase (bcc) has a degeneracy of only 1, and the other 1-phases (CP), as in our model, have a twofold degeneracy. With the described physical picture in mind it is straightforward to generalize the degeneracy of the 0 -phase to any value $p \geqslant 1$. The parameter $p$ effectively describes the larger number of vibrational degrees 

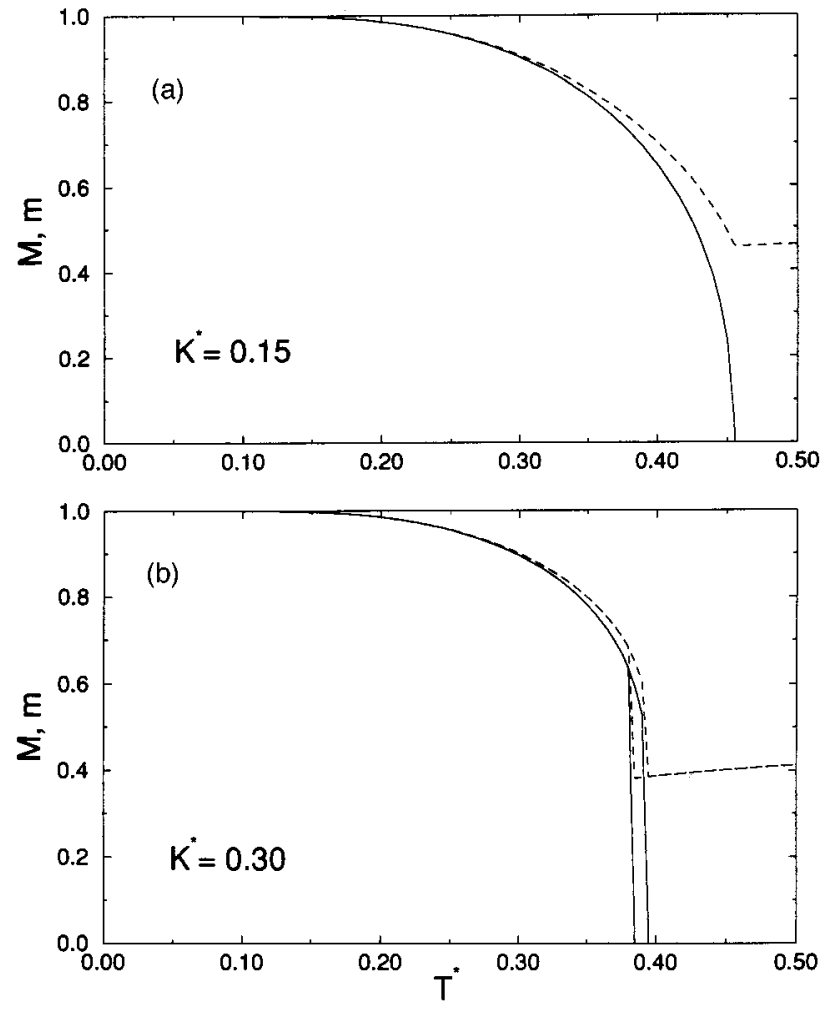

FIG. 1. Order parameter behavior as a function of $T^{*}$ for $p=2$ and two different values of $K^{*}$ : (a) $K^{*}=0.15$ and (b) $K^{*}=0.30$. The tricritical point is at $\left(T_{t}^{*}, K_{t}^{*}\right)=(0.4219,0.2298)$. Dashed lines correspond to $M$ and solid lines to $m$. One observes hysteresis effects at the first-order transition.

of freedom in the high-temperature phase.

The BEG model, or variants thereof, has often been used to describe the martensitic transformation. We wish to mention that Yamada and co-workers ${ }^{19}$ have discussed a pseudospin model which is a simplified case of the original BEG model for the martensitic transformation with respect to precursor effects. Goicoechea and Ortín ${ }^{20}$ have also used a BEG model but without including the possibility $p>1$ and restricted to $T=0$. We argue that the BEG model lacks an important part of physics involving the neglected, particularly large vibrational entropy of the b.c.c phase. Another and more pragmatic argumentation for our proposed generalization of the model for the present purpose is thus that an effect is needed which can add entropy to the high-temperature phase in a self-consistent manner. In terms of computer efficiency and simplicity the adding of a formal higher degeneracy to the phase representing the bcc phase is very advantageous.

The model turns out to be interesting with several surprising, systematic properties occurring as an additional dimension of the original BEG model. We shall call this model the degenerate BEG (DBEG) model. The main points are (i) the bcc phase is stabilized for increasing values of $p$, (ii) the first-order transition region is increased for increasing $p$, and (iii) the entropy change at the first-order transition is found to be independent of the transition temperature, although the latter is strongly dependent on the model parameters. Point (iii) has been found experimentally for the martensitic transformation in $\mathrm{Cu}$-based shape memory alloys, where the tran-

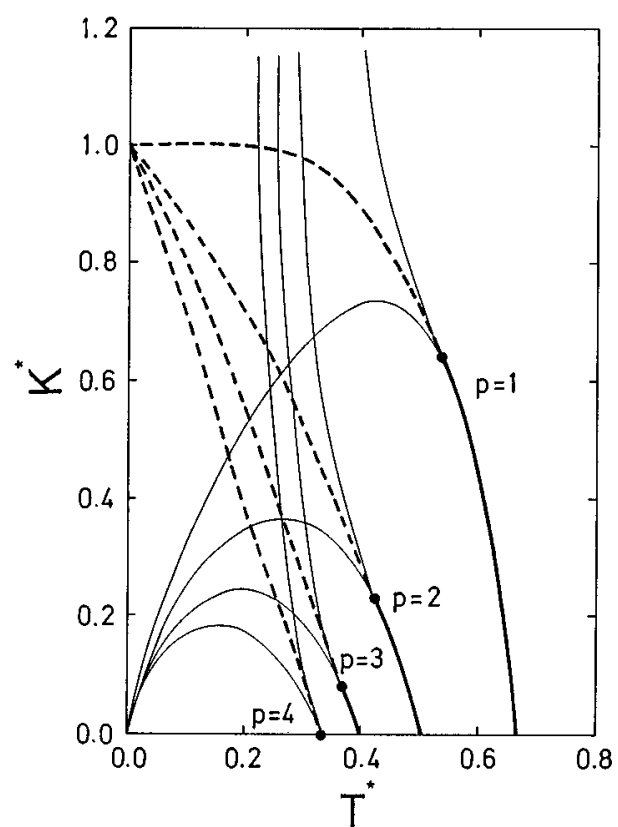

FIG. 2. Mean-field phase diagram for the DBEG model for $p=1,2,3$, and 4. Thick lines indicate equilibrium first-order (dashed line) and second-order (solid line) phase transitions between the bcc $(m=0)$ and the martensitic $(m \neq 0)$ phases. Thin lines represent the metastability limits (spinodal lines) of both phases.

sition temperature can be strongly varied by introducing small amounts of impurities. ${ }^{16}$

The DBEG model contains the $(q=p+2)$-state Potts model for particular values of the interaction parameters. However, we shall not pursue that less natural relation further. The main motivation for deriving the DBEG model is to find a simple model which shows a discontinuous transition between ordered phases as a function of temperature. This is a characteristic feature of the martensitic transformation. Thus one can study hysteresis effects as a function of temperature. Recently, hysteresis effects have been discussed in terms of a spin-glass model. ${ }^{17}$ Although the analogy between the spin-glass and martensitic problems, pointed out by Kartha et al., ${ }^{18}$ leads to interesting results, it is not completely clear how close that model is to the martensitic problem. In particular, the driving force for the spin-glass transition is a magnetic, external field instead of the temperature, which is not a field. In a subsequent paper we plan to study the effect of disorder on the DBEG model.

The paper is organized as follows. In Sec. II, we introduce the simplified and generalized version of the original LM model and show that it can be mapped onto the three-state BEG model. In Sec. III we solve and discuss the resulting DBEG model using the mean-field approximation (Sec. III A) and Monte Carlo techniques (Sec. III B). Finally, in Sec. IV we discuss the relation with the martensitic transformation problem and conclude.

\section{THE DBEG MODEL}

The minimal model for describing a transition between an energy-stabilized low-temperature state and an entropy- 
stabilized high-temperature ordered state is found to be governed by the Hamiltonian derived in the Appendix,

$$
\mathscr{H}=-\sum_{\langle i j\rangle}\left\{K S_{i z} S_{j z}+J S_{i x} S_{j x}\right\} .
$$

The spin variables are discrete, with values $S_{i x}= \pm 1$, and $S_{i z}=+1$ with a degeneracy factor $p \geqslant 1$. The physical reasoning behind this Hamiltonian and the degeneracy factor $p \neq 1$ is outlined in the Appendix. We redefine the state $S_{i z}=1$ as a state $\sigma_{i}=0$ and the states $S_{i x}= \pm 1$ as the states $\sigma_{i}= \pm 1$, which are the states in the BEG model. ${ }^{13}$ Then Eq. (2.1) becomes

$$
\mathscr{H}=-\sum_{\langle i j\rangle}\left\{K\left(1-\sigma_{i}^{2}\right)\left(1-\sigma_{j}^{2}\right)+J \sigma_{i} \sigma_{j}\right\},
$$

where the state $\sigma_{i}=0$ has degeneracy $p$. By expanding the first term one gets

$$
\mathscr{H}=-J \sum_{\langle i j\rangle} \sigma_{i} \sigma_{j}-K \sum_{\langle i j\rangle} \sigma_{i}^{2} \sigma_{j}^{2}+z K \sum_{i} \sigma_{i}^{2}-z K N / 2,
$$

where $z$ is the number of nearest neighbors and $N$ is the number of points on the lattice. Renormalizing the parameters, the Hamiltonian can be written in terms of just one parameter $K^{*}=K / J$. We shall restrict ourselves in this paper to the case $K^{*} \geqslant 0$. Consequently, Eq. (2.1) is exactly mapped onto a particular case of the degenerate BlumeEmery-Griffiths model, which can be written

$$
\begin{aligned}
\mathscr{H}^{*}= & \mathscr{H} \mid J=-\sum_{\langle i j\rangle} \sigma_{i} \sigma_{j}-K^{*} \sum_{\langle i j\rangle} \sigma_{i}^{2} \sigma_{j}^{2}+z K^{*} \sum_{i} \sigma_{i}^{2} \\
& -z K^{*} N / 2 .
\end{aligned}
$$

The ground state is one of the phases with either $\sigma_{i}=1$ or $\sigma_{i}=-1$ if $K^{*}<1$ and the phase with $\sigma_{i}=0$ if $K^{*}>1$. This can, of course, be also seen directly from Eq. (2.1). However, since the BEG model is standard in statistical physics, we will follow the notation of Eq. (2.4) in the remainder of this paper.

\section{PHASE DIAGRAM OF THE DBEG MODEL}

In this section we obtain the phase diagram of the DBEG model [Eq. 2.4] by using first a mean-field approximation and then Monte Carlo simulation techniques. The DBEG model is very suitable for analysis because it has only two parameters $K^{*}$ and $p$, limiting the space to explore in order to get the phase diagram. This together with the discrete character of the variables makes it ideal for computer simulation. However, to obtain an overall physical picture it is useful to start with the mean-field analysis. In addition, this can provide an analytical expression for the entropy change at the transition, which is an important experimental quantity not easily obtainable from Monte Carlo simulations.

\section{A. Mean-field solution}

It is convenient to define the following two order parameters:

$$
\begin{gathered}
m \equiv \sum \sigma_{i} / N=\left(N_{1}-N_{-1}\right) / N, \\
M \equiv \sum \sigma_{i}^{2} / N=1-N_{0} / N,
\end{gathered}
$$

where $N_{\sigma}$ is the number of points in the state $\sigma=\{+1,-1,0\}$. The entropy of the system can be written as

$$
S_{\mathrm{MF}}=k_{B} \ln \left(\frac{N !}{N_{1} ! N_{-1} ! N_{0} !} p^{N_{0}}\right),
$$

which yields to the following expression for the mean-field free energy per particle:

$$
\begin{aligned}
\frac{F_{\mathrm{MF}}^{*}}{N}=\frac{F_{\mathrm{MF}}}{z J N}=\frac{E_{\mathrm{MF}}^{*}}{N}-T^{*} \frac{S}{N k_{B}}= & -\frac{1}{2}\left(m^{2}+K^{*} M^{2}-2 K^{*} M\right)-\frac{K^{*}}{2}+\frac{T^{*}}{2}\left[(M+m) \ln \left(\frac{M+m}{2}\right)\right. \\
& \left.+(M-m) \ln \left(\frac{M-m}{2}\right)+2(1-M) \ln (1-M)-2(1-M) \ln p\right],
\end{aligned}
$$

where $T^{*}=k_{B} T / z J$ is the temperature in units of $z J / k_{B}$ (we shall in general let an asterisk (*) indicate this unit). Standard minimization of Eq. (3.4) gives the following two coupled equations for the temperature dependence of the order parameters $m$ and $M$ :

$$
M=m \operatorname{coth} \frac{m}{T^{*}},
$$

$$
2 K^{*}(M-1)=T^{*} \ln \left(p 2 \frac{(M+m)(M-m)}{4(1-M)^{2}}\right) .
$$

There exist two possible solutions: the phase with $m=0$ (bcc), which is stable for large values of $T^{*}$ and $K^{*}$, and the phase with $m \neq 0(\mathrm{CP})$, which is stable for low values of $T^{*}$ and $K^{*}$. The phases are separated by a transition line which can be of either first or second order. The first-order transition temperature $T_{0}^{*}\left(K^{*}\right)$ is found by equality of the free energies of the two phases. Figure 1 shows two ex- 
amples, for $p=2$, of the dependence of the order parameters $m$ and $M$ with $T^{*}$ for two different values of $K^{*}$. The change from a second-order [Fig. 1(a)] to a first-order [Fig. 1(b)] transition indicates the existence of a tricritical point. To determine it analytically we perform a Landau expansion in $m$ and $M_{z}=M-M_{c}$, where $M_{c}$ is the value of $M$ at the transition point. The expansion contains, beside the usual even terms, a term of the form $M_{z} m^{2}$. A term of this kind was discussed in connection with the martensitic transformation by the introduction of the LM model. ${ }^{9}$ Its presence shows the importance of two coupled strains, and it implies that the transitions need not be accompanied by mode softening. These generic features are thus preserved in the present highly simplified model. By eliminating $m_{z}$ [substituting (3.5) into (3.4)] we may write the Landau expansion in terms of the variable $m$ around $m=0$ as $f \sim f_{0}\left(T^{*}, K^{*}\right)+a\left(T^{*}, K^{*}\right) m^{2}+b\left(T^{*}, K^{*}\right) m^{4}+c\left(T^{*}, K^{*}\right)$ $\times m^{6}$. In the second-order region the transition temperature is given by the condition $a\left(T_{c}^{*}, K^{*}\right)=0$, which gives the relation

$$
\ln \frac{2\left(1-T_{c}^{*}\right)}{p T_{c}^{*}}=K^{*} \frac{1-T_{c}^{*}}{T_{c}^{*}} .
$$

In the first-order transition region this equation gives the spinodal line separating the regions for different growth mechanisms. The tricritical point is obtained by imposing simultaneously the conditions $a\left(T_{t}^{*}, K_{t}^{*}\right)=0$ and $b\left(T_{t}^{*}, K_{t}^{*}\right)=0$, leading to

$$
K_{t}^{*}=\frac{3 T_{t}^{*}-1}{2\left(1-T_{t}^{*}\right)}
$$

The complete phase diagram is presented in Fig. 2 for $p=1,2,3$, and 4 . A thick solid line indicates a secondorder transition whereas a thick dashed line represents a firstorder transition. Thin lines represent the metastability limits (or spinodal lines) of the two phases. For $K^{*}=0$ the critical temperature is $T_{c}^{*}=2 /(p+2)$. It is interesting that the model can describe the existence of two different kinds of growth of martensitic phase: (i) spontaneous (exponential) growth for $K^{*}>K_{t}^{*}$ for "internal" quenches to temperatures inside the lower spinodal line $\left[a\left(T_{c}^{*}, K^{*}\right)=0\right]$ and (ii) nucleation growth for either very deep or very shallow "external" quenches. The relative size of the region of exponential growth increases for increasing $p$. The influence of the degeneracy parameter $p$ is quite dramatic. First, increasing $p$ leads to an increasing range of values of $K^{*}$ for which the transition is first order. Second, for $p \geqslant 4$ there is no secondorder transition in the mean-field approximation.

We have also calculated the entropy change $\Delta S / N k_{B}$ at the transition point $T_{0}^{*}\left(K^{*}\right)$ for different values of $p$. The numerical results are shown in Fig. 3. It is interesting to note the existence of a plateau with constant $\Delta S \sim \ln p$ for a large region of $T^{*}$. This comes from the fact that the transition line for low values of $T_{0}^{*}$ exhibits a linear behavior, $T_{0}^{*}$ $\propto\left(1-K^{*}\right)$, exactly as the dependence of the difference in internal energy between the two phases, $\Delta E^{*} \propto\left(1-K^{*}\right)$. To understand this behavior it is convenient to rewrite the selfconsistent equations (3.5) and (3.6) for the order parameters in energy-level form. For the $m=0$ phase one finds

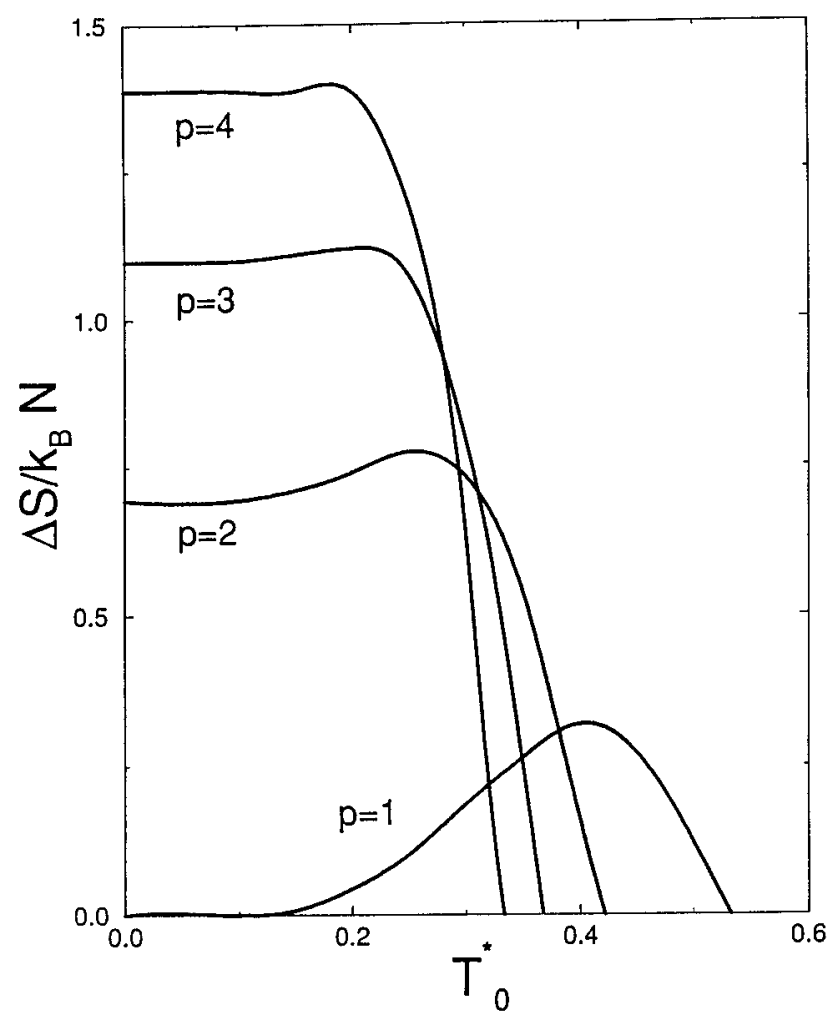

FIG. 3. Mean-field entropy change at the transition for different values of the first-order transition temperature, $T_{0}^{*}\left(K^{*}\right)$, for $p=1$, 2,3 , and 4 .

$$
1-M=\frac{p e^{K^{*}(1-M) / T^{*}}}{p e^{K^{*}(1-M) / T^{*}}+2} .
$$

This directly shows that $M \rightarrow 0$ exponentially for low temperatures. For the $m \neq 0$ phase one gets

$$
\begin{gathered}
1-M=\frac{p e^{K^{*}(1-M) / T^{*}}}{p e^{K^{*}(1-M) / T^{*}}+e^{m / T^{*}}+e^{-m / T^{*}}}, \\
m=\frac{e^{m / T^{*}}-e^{-m / T^{*}}}{p e^{K^{*}(1-M) / T^{*}}+e^{m / T^{*}}+e^{-m / T^{*}}} .
\end{gathered}
$$

This shows that for low temperatures both $m$ and $M$ tend exponentially to 1 . By expanding at low temperatures we find the entropy change at the transition line

$$
\frac{\Delta S}{N k_{B}}=\ln p+\frac{2}{p} e^{-K^{*} / T^{*}}\left[1+\frac{K^{*}}{T^{*}}\right]-p e^{-1 / T^{*}}\left[1+\frac{1}{T^{*}}\right] .
$$

Accordingly, for $K^{*}<1$ and small $T^{*}$, we find that $\Delta S$ shows an exponentially weak increase as a function of $T^{*}$ before tending to zero at the tricritical point. The implications of this plateau in relation to martensitic transformations will be discussed later.

\section{B. Monte Carlo simulation}

The standard Metropolis algorithm for Monte Carlo simulations has been used to solve numerically the DBEG model. 


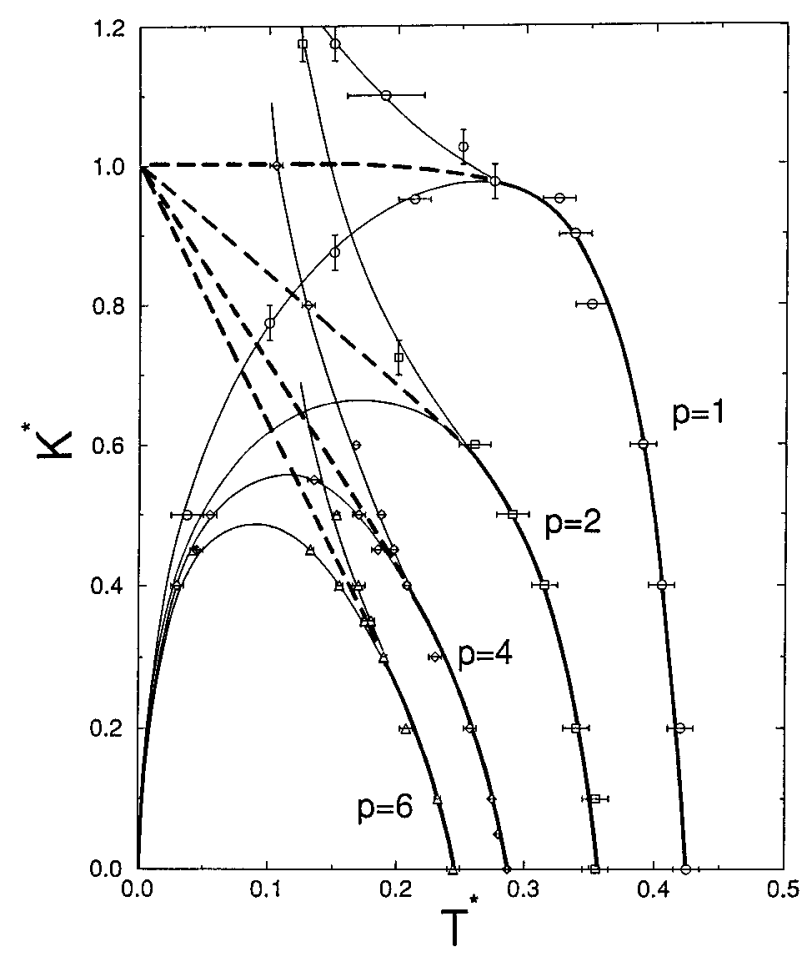

FIG. 4. Phase diagrams for $p=1,2,4$, and 6 obtained from Monte Carlo simulations for a $64 \times 64$ lattice. Lines are guides to the eye indicating first-order transition (thick dashed line), secondorder transition (thick solid line), and spinodal (thin line) lines.

We have used a vectorial algorithm on an IBM3090 supercomputer. We have restricted to the study of the twodimensional (2D) square lattice for cases $p=1,2,4$, and 6 . The results have been obtained on a $64 \times 64$ lattice and are averaged over $10^{4}$ Monte Carlo steps per system (MCS). We have located the phase transitions on the $\left(T^{*}, K^{*}\right)$ plane by calculating the averaged order parameters $\langle|m|\rangle$ and $\langle M\rangle$. The metastability limits have been estimated by starting the simulations from a configuration equilibrated at one side or another of the transition line and changing (in small steps lasting for $10^{3} \mathrm{MCS}$ ) the parameters $T^{*}$ and $K^{*}$ until the jump to the new phase is observed. This has been possible due to the difference in the growth mechanism mentioned before. The phase diagrams for $p=1,2,4$, and 6 are shown in Fig. 4. The temperature dependence of the simulated order parameters is found to be similar to the mean-field behavior. The entropy change given by Eq. (3.12) is exact for $T=0$. Accordingly, we can deduce that the entropy change at the transition simulated by the Monte Carlo method is almost identical to the mean-field results (Fig. 3) at low temperatures. It was found in a detailed study of entropy effects in a modified LM model ${ }^{21}$ that the entropy obtained by meanfield theory and by Monte Carlo simulation is in good agreement for $T$ not too close to $T_{c}$.

In Fig. 4 it is consistent with the data to assume that the first-order transition temperature follows $T_{0}^{*}\left(K^{*}\right)$ $\propto\left(1-K^{*}\right)$ at low temperatures, as in the mean-field case. We notice that for $p=1$ (the BEG model) the fluctuations renormalize the tricritical point to $K^{*} \simeq 1$. Therefore it is very difficult to cross the first-order line by varying the temperature. This problem is not present in the mean-field phase diagram. This shows that it is important to allow larger values of $p$ since, in this case, one can cross the first-order line for a large range of $K^{*}$ values. Further, for larger values of $p$, the maximum in the lower spinodal line occurs at higher values of $K^{*}$ than in the mean-field case. It is higher than the tricritical point, thus allowing for the two different growth mechanisms after quenches as discussed. The tricritical point remains in the positive region of $K^{*}$ at least up to $p=6$, contrary to the mean-field approximation.

\section{DISCUSSION AND CONCLUSION}

We have introduced a model which shows both a firstorder and a second-order transition between two ordered phases as a function of temperature. The model exhibits a strong dependence of the transition temperature as a function of the only energy parameter $K^{*}$. The high-temperature phase is stabilized by an entropic part controlled by a degeneracy factor $p$. In the standard Blume-Emery-Griffiths model one has $p=1$, but we have generalized this to higher values with the intention of including additional entropy in a phase due to a richer space for vibrational degrees of freedom. In the simplest case $p$ is an integer, but it may also be thought of as having any value $p \geqslant 1$. For increasing values of $p$ the region of stability of the high-temperature phase is increased both in $K^{*}$ and $T^{*}$ space. Further, the first-order transition region is increased. On the other hand the entropy change across the transition line exhibits a plateau with almost no temperature dependence.

The model was introduced in order to describe the pertinent features of martensitic transitions. For these, it is known ${ }^{6}$ that there is a low-energy valley in the phonon spectrum with corresponding high density of states. Although no mode goes completely soft, this plays an important role in the transition. In our model this feature has been simplified by discrete energy levels with a temperature dependence of the effective frequencies given by the self-consistent temperature dependence of the order parameters. This leads to the entropy plateau. In the presence of impurities the bcc phase shows static deformations, tweed patterns. Only after a spatial average is the simple bcc phase obtained. The excess "configurational" entropy due to this deformation is well described by the degeneracy factor $p$. It has in fact been observed, ${ }^{16}$ but not understood, that the martensitic transition in $\mathrm{Cu}$-based shape memory alloys takes place at a point where the entropy change assumes a characteristic value $\left(\Delta S / k_{B} N \sim 0.2\right)$, although the transition temperature may vary strongly due to alloying with small amounts of impurities. This alloying does not modify the symmetry of the problem, and so we expect that the vibrationalconfigurational entropy excess is the same irrespective of the concentration. That means the value of $p$ is independent of concentration. On the other hand the local forces, in the model represented by $K^{*}$, are strongly modified. Thus, for fixed $p$, the introduction of impurities corresponds to varying $K^{*}$, which does not influence the entropy change. One may therefore conclude either that our model gives a first explanation of the observed constant entropy change criterion ${ }^{16}$ or that these measurements do support our proposed simple way of including the complicated vibrational entropy, expected to be essential in stabilizing the bcc phase. A second 
important element of real martensitic transformations in impure systems is that they exhibit a coexistence region where, for a given temperature, given amounts of both phases are in equilibrium. When the temperature is changed the transition proceeds spontaneously to a new equilibrium. We expect this is associated with the impurity influence on the forces, which would give rise to an inhomogeneous distribution of transition temperatures. This phenomenon is not observed in pure systems like $\mathrm{Zr}^{6}{ }^{6}$ By extending the model to include randomness we expect to be able to describe the transition region characteristic for conventional shape memory alloys.

\section{ACKNOWLEDGMENTS}

We acknowledge supercomputing support from Fundació Catalana per la Recerca (FCR) and Centre de Supercomputació de Catalunya (CESCA), and financial support from DGCyT (Spain), Project No. MAT92-0884. P.-A.L. acknowledges DGCyT (Spain) for financial support (Grant No. SAB0036). We thank H. Richards for linguistic comments.

\section{APPENDIX: BASIS AND SIMPLIFICATION OF THE LM MODEL}

The LM model was introduced by Lindgård and Mouritsen ${ }^{9}$ in order to simulate the coupling between an internal shuffle strain and a uniform strain. These strains were identified as the most important agents for the martensitic transformation in a model case like the hcp $\rightarrow$ bcc transformation occurring in $\mathrm{Zr}$. The atomic positions within a unit cell were mapped onto continuous spin variables. Only the projection onto a plane was considered, thus reducing the problem to a two-dimensional one. The price one pays for this is that the model no longer contains the blocking effect which is important in the presence of conflicting long-range acting uniform strains. The LM model thus simplifies the real martensitic problem by only considering four nonconflicting variants of the hcp phase. The bcc phase is represented by a ferromagnetic phase with the spins pointing in the $z$ direction out of the plane of a square mesh corresponding to the projection onto the $\langle 110\rangle$ bcc plane. The shuffle mode, shuffling every second plane, $\langle 1 \overline{1} 0\rangle$, in the bcc structure is needed in order to produce the hcp structure. This can be represented in the projection by the spins ordering antiferromagnetically, in antiparallel chains in the projection plane, in either the $\pm x$ or $\pm y$ direction, corresponding to four variants (described by the $J$ term below). Because of the projections, we call the considered states hcp and bcc in the following.

In a more complete paper ${ }^{10}$ the model was further analyzed using advanced spin-wave theory. It was demonstrated that the model described the atomic motion in the unit cell with the exception of the freedom to move in the energetically most unfavorable direction. The free energy and the contribution from the vibrational entropy were discussed. It was found that in a quasiharmonic theory, the vibrational entropy does not play a dominant role for the model. The Monte Carlo simulations include those effects more faithfully. The continuous, two-dimensional LM spin model is described by the Hamiltonian

$$
\begin{aligned}
\mathscr{H}_{\mathrm{LM}}= & \sum_{\langle i j\rangle}\left\{-K S_{i z} S_{j z}+J\left[\mathbf{S}_{i} \cdot \mathbf{S}_{j}-P\left(\mathbf{r}_{i j} \cdot \mathbf{S}_{i}\right)\left(\mathbf{r}_{i j} \cdot \mathbf{S}_{j}\right)\right]\right\} \\
& -D \sum_{i}\left(S_{i x}^{4}+S_{j x}^{4}\right)
\end{aligned}
$$

The parameters $K>0$ and $J>0$ favor the bcc and hcp phases, respectively. The $J$ term is obviously of the dipole form and by the parameter $P$ (usually equal to 3 ) we can vary the interface energy for domain boundaries between the variants. Finally, the $D$ term was introduced in order to avoid the principal lack of order in two dimensions for the Heisenberg-type continuous spin models. It restricts the spins in the hcp phase to lie predominantly in the $\pm x$ or the $\pm y$ direction. This term is in fact important for the description of the transition at a given temperature $T_{M}$ for a fixed ratio $K / J$, since it causes the first-order phase separation line to curve as a function of temperature, stabilizing the bcc phase at high temperatures. This is due to the higher powers of the involved spin operators in that term than in the second-order interaction terms. A modified version of the model was used $^{21}$ in which the $K$ term was replaced by a single-site field term for describing the transition from a ferromagnetically polarized (disordered) state to an antiferromagnetic state. The phase separation line shows a tricritical point, separating a discontinuous transition at high fields and at low temperatures from a continuous transition at low fields and at higher temperatures. Below we shall restrict the $z$ component $S_{i z}$ of the spins to positive values. This makes the problem similar to the just-mentioned field problem, although the $K$ term then represents a self-consistent, internal field. In the papers by Castán and Lindgård the model was studied with respect to domain growth kinetics ${ }^{11}$ and pinning properties. ${ }^{12}$ Here it became clear that the model could be simplified considerably without changing the most fundamental goal, the description of a transition between a bcc and a hcp phase. However, the domain growth kinetics and the pinning properties are strongly affected by simplifications. With the aim of constructing a minimal model for the martensitic transformation, without internal pinning properties, we shall now simplify the model even further.

First of all, we let $D \rightarrow \infty$ which is equivalent to considering only a discrete spin model. By doing this the model no longer describes the vibrational entropy. Second, it is not necessary to consider an antiferromagnetic order for representing the hcp phase, since we can formally reverse every second chain. One then gets domains of the simpler ferromagnetic structure, without loss in the description of the domain properties. Let us therefore replace the dipole term by a simple Ising term favoring a ferromagnetic order in the plane along $x$ or $-x$. Finally, we let the bcc phase be represented by only one variable $+z$. The presence of the possibility $-z$ in Eq. (A1) is somewhat artificial, but it allows a transition from the bcc phase to a disordered one at high temperatures (corresponding to the melting transition). Since we are not interested in that, it is preferential to consider only the state $+z$. We have now reduced $\mathscr{H}_{\mathrm{LM}}$ to just the first two terms with discrete variables [i.e., eliminating the $D$ and $P$ terms in Eq. (A1)]. Let us now, in addition, allow that the system can adopt the state $+z$ with an arbitrary high probability, corresponding to a higher degeneracy. This adds a 
self-consistent entropy to the high-temperature phase, which mimics the now neglected vibrational entropy in a general way. This entropy is very difficult to calculate in a realistic model, since it includes the ordinary quasiharmonic phonon contribution as well as the effects due to their damping and from the soft mode and central peak phenomena. For the purpose of simplicity in modeling this, we give the $+z$ phase the degeneracy number $p \geqslant 1$. For the low-temperature phases, on the other hand, we only consider two variants, say, $\pm x$, since anyway the domain wall properties between the different variants are not faithfully described by the model after the strong simplifications. Finally, we have reduced Eq. (A1) to a Hamiltonian $\mathscr{H}$ involving only three states, one of which has an arbitrary degeneracy $p \geqslant 1$,

$$
\mathscr{H}=-\sum_{\langle i j\rangle}\left\{K S_{i z} S_{j z}+J S_{i x} S_{j x}\right\}
$$

In this we have for simplicity used the opposite sign for $J$ than in Eq. (A1). This Hamiltonian is analyzed in the main part of the paper.
${ }^{1}$ Z. Nishiyama, Martensitic Transformations (Academic Press, New York, 1978); Phase Transformations in Solids, MRS Symposia Proceedings No. 21, edited by T. Tsakalakos (NorthHolland, New York, 1984); European Symposium on Martensitic Transformations and Shape Memory Properties, edited by G. Guénin [J. Phys. (Paris) IV Colloq. 1, C4 (1991)]; A. G. Khachaturyan, Theory of Structural Transformations in Solids (Wiley, New York, 1983).

${ }^{2}$ C. Zener, Phys. Rev. 71, 846 (1947).

${ }^{3}$ J. Friedel, J. Phys. Lett. (Paris) 35, L59 (1974).

${ }^{4}$ R. E. Watson and M. Weinert, Phys. Rev. B 30, 1641 (1984).

${ }^{5}$ Y.-Y. Ye, Y. Chen, K.-M. Ho, B. N. Harmon, and P.-A. Lindgård, Phys. Rev. Lett. 58, 1769 (1987).

${ }^{6}$ W. Petry, A. Heiming, J. Trampeneau, M. Alba, C. Herzig, H. R. Schober, and G. Vogl, Phys. Rev. B 43, 10933 (1991; 43, 10948 (1991).

${ }^{7}$ L. Mañosa, A. Planes, J. Ortín, and B. Martínez, Phys. Rev. B 48, 3611 (1993).

${ }^{8}$ P.-A. Lindgård, J. Phys. (Paris) IV Colloq. C4-3 (1991).

${ }^{9}$ P.-A. Lindgård and O. G. Mouritsen, Phys. Rev. Lett. 50, 690
(1986).

${ }^{10}$ P.-A. Lindgård and O. G. Mouritsen, Phys. Rev. B 41, 688 (1990).

${ }^{11}$ T. Castán and P.-A. Lindgård, Phys. Rev. B 40, 5069 (1989).

${ }^{12}$ T. Castán and P.-A. Lindgård, Phys. Rev. B 43, 956 (1991).

${ }^{13}$ M. Blume, V. J. Emery, and R. B. Griffiths, Phys. Rev. A 4, 1071 (1971).

${ }^{14}$ J. R. Morris and R. J. Gooding, Phys. Rev. Lett. 65, 1769 (1990).

${ }^{15}$ J. R. Morris and K.-M. Ho, Phys. Rev. Lett. 74, 940 (1995).

${ }^{16}$ A. Planes, L. Mañosa, D. Rios-Jara, and J. Ortín, Phys. Rev. B 45, 7633 (1992).

${ }^{17}$ J. P. Sethna, K. Dahmen, S. Kartha, J. A. Krumhansl, B. W. Roberts, and J. D. Shore, Phys. Rev. Lett. 70, 3347 (1993).

${ }^{18}$ S. Kartha, T. Castán, J. A. Krumhansl, and J. P. Sethna, Phys. Rev. Lett. 67, 3630 (1991).

${ }^{19}$ K. Fuchiazaki, Y. Noda, and Y. Yamada, Phys. Rev. B 39, 9260 (1989); Y. Yamada and K. Fuchiazaki, ibid. 42, 9420 (1990).

${ }^{20}$ J. Goicoechea and J. Ortín, J. Phys. (France) IV Colloq. 5, C2-71 (1995).

${ }^{21}$ P.-A. Lindgård, H. E. Virtiö, and O. G. Mouritsen, Phys. Rev. B 38, 6798 (1988). 